\title{
EchoGéo
}

$54 \mid 2020$

Varia

\section{Se nourrir par l'agriculture périurbaine à São Paulo}

Enjeux socio-spatiaux d'un système alimentaire métropolitain

Feeding yourself through peri-urban farming in São Paulo. Socio-spatial challenges of a metropolitan food system

\section{Angèle Proust}

\section{OpenEdition}

\section{Journals}

\section{Édition électronique}

URL : https://journals.openedition.org/echogeo/20585

DOI : $10.4000 /$ echogeo.20585

ISSN : 1963-1197

\section{Éditeur}

Pôle de recherche pour l'organisation et la diffusion de l'information géographique (CNRS UMR 8586)

\section{Référence électronique}

Angèle Proust, « Se nourrir par l'agriculture périurbaine à São Paulo », EchoGéo [En ligne], 54 | 2020, mis en ligne le 31 décembre 2020, consulté le 26 août 2021. URL : http://journals.openedition.org/ echogeo/20585 ; DOI : https://doi.org/10.4000/echogeo.20585

Ce document a été généré automatiquement le 26 août 2021.

EchoGéo est mis à disposition selon les termes de la licence Creative Commons Attribution - Pas d'Utilisation Commerciale - Pas de Modification 4.0 International (CC BY-NC-ND) 


\title{
Se nourrir par l'agriculture périurbaine à São Paulo
}

\author{
Enjeux socio-spatiaux d'un système alimentaire métropolitain \\ Feeding yourself through peri-urban farming in São Paulo. Socio-spatial \\ challenges of a metropolitan food system
}

Angèle Proust

\section{Introduction}

1 La Région Métropolitaine de São Paulo (RMSP) ${ }^{1}$, plus grande agglomération d'Amérique latine avec 21,3 millions d'habitants est confrontée à un grand nombre de défis, dont celui de nourrir sa population. Le système alimentaire, qui va de la production à la consommation, joue un rôle politique révélateur des mécanismes de construction des inégalités socio-spatiales sur le territoire. Les population marginalisées et défavorisées n'ont pas accès à une alimentation saine et de qualité, que ce soit par manque de structure d'approvisionnement ou à cause de leur précarité. Pour répondre à ces défaillances, des agriculteurs familiaux s'organisent pour produire des aliments au sein même du tissu urbain tentaculaire de la métropole brésilienne. Le plus souvent, c'est une stratégie d'auto-subsistance qui motive leur démarche, mais il arrive aussi que se dessinent des circuits économiques informels autour des producteurs. Ceux-ci alimentent alors les quartiers les plus pauvres et contribuent à mettre en place un « vivrier marchand » dans les marges de la métropole (Chaléard et al., 1996). Nombreux sont les obstacles à cette agriculture périurbaine, à commencer par le déploiement de l'habitat d'auto-construction qui accentue la pression foncière sur les espaces vacants. Le front d'urbanisation met en péril le maintien d'une agriculture ancienne et marginale qui constitue par ailleurs un atout politique d'émancipation des acteurs locaux.

2 Au potentiel politique et symbolique de l'agriculture périurbaine pour l'autonomisation des marges urbaines s'ajoutent ses capacités concrètes en termes d'approvisionnement 
alimentaire. Cet aspect diffère en tout point de l'agriculture urbaine qui se répand dans les quartiers centraux les plus aisés. Celle-ci répond à des fonctions socioenvironnementales et relève en réalité d'une forme de jardinage urbain permettant de reverdir la métropole. Or, dans une cité où la majorité des habitants n'a pas accès à des aliments en qualité ni même en quantité suffisante, les fonctions productives et alimentaires de l'agriculture urbaine sont à privilégier pour permettre de renforcer le système alimentaire. L'agriculture périurbaine, qui associe fonction alimentaire et intégration des marges, contribue de manière plurielle à la justice alimentaire sur le territoire. Quel est le potentiel de la production agricole périurbaine pour approvisionner la métropole depuis ses marges et faire exister des circuits-courts intraurbains qui répondent aux besoins des citadins les plus précaires ? Ces réserves nourricières sont-elles à même de satisfaire les besoins alimentaires de la métropole de São Paulo?

Il s'agit de comprendre en quoi l'agriculture périurbaine constitue un levier essentiel pour la justice alimentaire à l'échelle métropolitaine, dont les besoins ne sont actuellement pas pris en compte par les politiques publiques en faveur de l'agriculture et du jardinage urbain, celles-ci privilégiant leurs fonctions socio-environnementales. Ces questions sont d'autant plus actuelles que le nouveau Plan Directeur Stratégique de São Paulo édité en 2014 reconnaît pour la première fois la présence de zones rurales à vocation agricole dans des quartiers périphériques de la ville. Cette avancée considérable pour la reconnaissance de l'agriculture périurbaine nous conduira d'abord à relever les mutations récentes du système alimentaire au regard des changements de paradigme relatifs à l'alimentation et du soutien à demi-teinte des politiques publiques. La dynamique territoriale observée dans les espaces urbains et périurbains y sera analysée comme facteur de maintien ou de fragilisation de l'agriculture à São Paulo. Enfin, la production alimentaire en milieu urbain sera appréhendée comme vecteur d'une démarginalisation des acteurs et des territoires par l'intermédiaire d'un processus de construction politico-identitaire.

\section{Des « systèmes alimentaires alternatifs » au prolongement du système classique}

\section{Un carrefour de commerce mondialisé en proie à des déséquilibres d'approvisionnement alimentaire}

4 L'approvisionnement alimentaire de la métropole de São Paulo est influencé par la présence, sur son territoire ou à proximité directe, de plusieurs infrastructures de commerce mondialisées. La Compagnie d'entrepôts et de magasins généraux de São Paulo (Ceagesp) est le plus grand marché alimentaire grossiste d'Amérique latine, qui représente environ $35 \%$ de la quantité totale de produits commercialisés dans les 26 centrales d'approvisionnement au Brésil (Wegner et Belik, 2012). Ce marché grossiste centralise et redistribue l'immense majorité des denrées consommées à São Paulo, mais il ne constitue pas un symbole de qualité pour les populations locales. Selon un acteur interrogé, en moyenne $30 \%$ des produits arrivent sur les étals déjà gâtés et sont jetés ${ }^{2}$. À 85 kilomètres de là, sur le littoral, se trouve le port de Santos, le plus grand point de passage des marchandises d'import-export du continent latinoaméricain. São Paulo est donc un point de contact majeur des denrées alimentaires et 
agricoles destinées à l'exportation, où transitent les marchandises issues de l'agrobusiness ${ }^{3}$. Dans l'État de São Paulo, les grandes exploitations prédominent en valeur de production sur les petites exploitations familiales, indiquant une orientation économique internationalisée et moins destinée au marché local (Tonneau, 2010).

L'agriculture productiviste est favorisée car elle représente un enjeu politico-financier pour les décideurs et gestionnaires. À l'inverse, l'agriculture familiale reçoit peu d'aide des pouvoirs publics à l'échelle fédérale alors même qu'elle fournit près de $70 \%$ de la demande domestique. Cependant, à des échelles de gouvernance infranationales, quelques politiques publiques ont entrainé la revalorisation des producteurs familiaux. Les agriculteurs périurbains en sont parfois les bénéficiaires, considérant l'ampleur du défi que représente le fait de nourrir São Paulo. Dans les années 1980, Franco Montoro, gouverneur de l'État de São Paulo (1983-1987), a lancé un programme de soutien à l'agriculture périurbaine, en encourageant la création de jardins et potagers sur des terrains appartenant à des entreprises. L'Eletropaulo, fournisseur d'électricité à São Paulo, a cédé un grand nombre de terrains situés sous des lignes à haute tension. La Petrobrasil qui distribue le pétrole et la Sabesp qui récupère les eaux usées ont également participé au projet. Celui-ci a permis de créer environ 10000 terrains un peu partout dans la métropole (Caldas et Jayo, 2019). À l'échelle municipale, la ville de São Paulo a rédigé en 2014 un nouveau Plan Directeur Stratégique sous la direction de Fernando Haddad (2013-2016). Ce plan a entrainé la reconnaissance de zones agricoles rurales et a permis l'identification des agriculteurs périurbains.

6 Ces logiques de légitimation de l'agriculture familiale en zone périurbaine posent les bases d'un nouveau paradigme agricole qui promeut la mise en place concrète d'un système alimentaire à l'échelle métropolitaine. Néanmoins, les tentatives de créer des réseaux complets de distribution manquent et le problème de l'accès aux produits persiste. Le programme Ligue os Pontos ${ }^{4}$ (2016-2020) a organisé la détection et la mise en cadastre des exploitations urbaines et périurbaines, non pas pour assurer le suivi technique et agronomique des agriculteurs, mais plutôt pour organiser la commercialisation de leurs produits avec le centre de São Paulo. Le souci de ce type de projet est, d'une part, le manque de durabilité dans le temps une fois le programme terminé et, d'autre part, la non prise en compte du caractère systémique du fait alimentaire. Les inégalités socio-spatiales font que ces circuits courts ne sont accessibles qu'aux populations dont le capital économique et culturel est conséquent. À São Paulo, une majorité de personnes vivent dans des " déserts alimentaires ", c'est-àdire des espaces urbains pauvres où les habitants ne peuvent pas se procurer des aliments sains à des prix abordables (Paddeu, 2012). Ils n'ont alors pas accès à ce type de produit, ce qui est problématique dans un pays où près de la moitié des adultes et un tiers des enfants souffrent de maladies nutritionnelles (diabète, obésité, maladies cardiaques) causées par une alimentation trop industrielle (Levasseur, 2017).

7 Le système agroalimentaire classique, omniprésent à São Paulo, permet à la métropole d'assurer son approvisionnement et sa sécurité alimentaire, ce qui n'assure en rien la qualité nutritionnelle des denrées ni leur équitable répartition. En conséquence, et compte tenu de la prédominance de la pauvreté et des inégalités au Brésil, les circuits alimentaires locaux adaptés à des critères de qualité discriminent les habitants plus qu'ils ne les approvisionnent. 


\section{D'un food movement " alternatif » à un mouvement de justice sociale, spatiale, alimentaire}

Pour favoriser la consommation de produits de meilleur qualité, l'émergence des "systèmes alimentaires alternatifs" prône une rupture avec le système agroalimentaire dominant et encourage à choisir des aliments frais, locaux et si possible «bio" plutôt que des aliments produits et transformés industriellement (Beischer et Corbett, 2016). Ces systèmes prennent comme point de départ les consommateurs en tant que moteurs d'une nouvelle démocratie alimentaire (Deverre et Lamine, 2010). Reflet du food movement majoritairement soutenus par les classes moyennes-supérieures, les systèmes alimentaires alternatifs adoptent une vision centrée sur le consommateur qui bouscule peu la structure d'approvisionnement classique et se contente de conforter le système alimentaire dominant (Paddeu, 2012).

C'est au travers d'une critique radicale qui incite à redonner un rôle central aux producteurs que la justice alimentaire propose de reconsidérer les systèmes alimentaires depuis leur base (Hochedez et Le Gall, 2016 ; Sbicca, 2018). Les travaux de Black food geographies ${ }^{5}$ appuient cette logique, avec pour objectif de transformer l'analyse du champ alimentaire et d'interroger la perception et l'expérience des populations racisées (Ramírez, 2015). Les petits agriculteurs périurbains à São Paulo, racisés et marginalisés, sont au croisement de ces deux cadres théoriques. Dans leur ensemble, les Noirs et les Latino-américains sont aussi les plus susceptibles d'habiter un " désert alimentaire » et d'être visés par les stratégies publicitaires des fast-food (Jones, 2019). Les agriculteurs périurbains luttent concrètement d'en bas contre cette réalité. L'agriculture périurbaine est un élément central de la justice alimentaire et porte en elle l'instauration d'une gouvernance alimentaire locale, définie comme l'articulation des politiques, des acteurs et des lieux permettant de faire dialoguer la ville, l'agriculture locale et l'alimentation des citadins (Perrin et Soulard, 2014).

10 Cependant, le manque de visibilité des agriculteurs périurbains limite la reconnaissance de leur engagement politique à une vision utilitariste. À São Paulo, la charge politique de l'agriculture périurbaine est devancée par celle du jardinage urbain qui se déploie dans les quartiers aisés et centraux comme une "guerilla gardening", concrétisant un rapprochement conceptuel entre agriculture urbaine et militantisme (Visoni et Nagib, 2019). Le jardinage urbain s'apparente à une lutte pacifique pour la reconquête du droit à la ville, qui concrétise l'appropriation de l'espace par la mise en culture de potagers communautaires par des jardiniers amateurs (Nagib, 2016). Le but de cette activité est d'abord de produire des discours et des idées avant de produire des aliments (Caldas et Jayo, 2019). Dans les faits, les jardins communautaires répondent aux préoccupations paysagères et récréatives d'une élite urbaine et participent à la diffusion du food movement au sein d'un microcosme socio-culturel relativement uniforme.

11 L'agriculture périurbaine, pratiquée par des populations marginalisées et racialisées au sein de marge socio-spatiales, suscite une vision critique du territoire. Qui a le droit de produire son alimentation au sein de la ville et où? La première fonction des jardins cultivés dans les quartiers centraux n'est pas alimentaire et pourtant leur visibilité est plus importante. À ce titre, les exploitations périphériques constituent une pratique à part et non pas une continuité de la conception récente d'une agriculture urbaine issue des pays des Nords. Les logiques productives de l'agriculture sont revisitées non 
seulement depuis les marges, mais aussi depuis les Suds, ce qui active de nouveaux modèles. Ce caractère essentiel de l'agriculture nous interpelle, conceptualisé depuis de nouveaux lieux et pour de nouveaux acteurs. À ce titre, les défis auxquels les agriculteurs périurbains sont confrontés mettent en évidence la mise en tension d'un système confronté à de récente mutations.

\section{Des dynamiques agricoles à l'œuvre dans les marges urbaines : le défi des périphéries}

\section{Inclure l'agriculture périurbaine aux circuits commerciaux métropolitains}

Les entretiens pratiqués à São Paulo ${ }^{6}$, alimentés par des observations distantes et participantes, ont permis de distinguer deux grandes catégories d'acteurs qui cultivent en ville: les agriculteurs périurbains et les jardiniers communautaires. Ces deux ensembles sont polarisés dans l'espace. La localisation des jardins au cœur de la tache urbaine est probante, tandis que les exploitations agricoles de subsistance sont principalement situées sur les périphéries en bordure de l'agglomération ${ }^{7}$.

Illustration 1 - La formation d'une ceinture périurbaine agricole et des jardins qui peuplent le cœur de l'agglomération

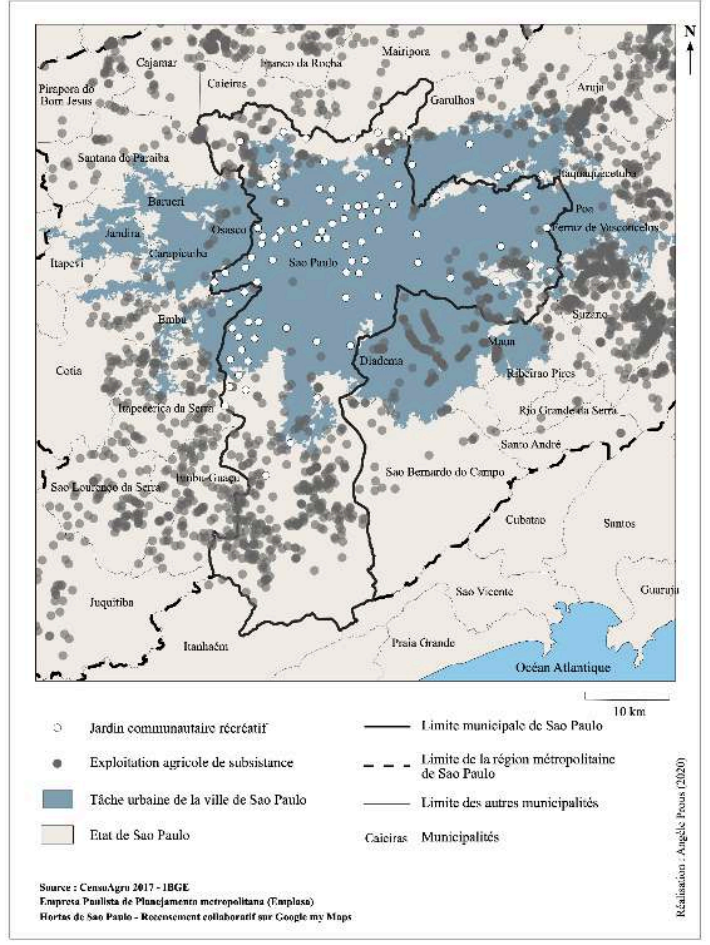

Les quartiers centraux de São Paulo, bien qu'ils puissent être perçus à certains égards comme des espaces productifs en raison de la présence de ces jardins communautaires, sont d'abord et avant tout des espaces de consommation. C'est ici que l'on retrouve la majorité des commerces alternatifs qui constituent le food movement pauliste, comme les marchés biologiques de plein vent, les points d'économie sociale et solidaire et les 
groupes de consommateurs responsables. Selon la Mapa de feiras orgânicas ${ }^{8}, 56$ lieux alimentaires du mouvement alternatif existent à São Paulo. La majorité des producteurs à la base de ces circuits sont des agriculteurs «bio » bien structurés qui cultivent dans la ceinture maraichère rurale autour de l'agglomération pauliste.

Il est en revanche plus rare de trouver des producteurs périurbains. Premièrement, parce que la plupart ne possèdent pas de certification biologique auditorada (vérifiée) délivrée par le ministère de l'Agriculture ou par l'Instituto Biodinâmico (IBD). Celle-ci, reconnue comme la seule certification authentique, coûte trop chère pour beaucoup de petits producteurs de la région métropolitaine qui ne possèdent souvent qu'une certification rotativa (rotative), laquelle ne leur donne pas le droit de recourir à un intermédiaire commercial pour vendre leurs produits. Cette certification dite alternative relève d'une Organisation de contrôle social (OCS) permettant aux agriculteurs d'une coopérative de se garantir entre eux en pratiquant des contrôles réguliers. Ce système fonctionne sur la base d'une confiance réciproque entre les distributeurs, les consommateurs et les producteurs; il est donc complètement gratuit et met à disposition une offre de produits sans intrants chimiques plus accessibles financièrement.

Très peu d'agriculteurs périurbains sont en mesure de vendre leurs produits comme du bio, puisqu'ils n'ont qu'une certification OCS. Certaines réponses ont pourtant été formulées du côté des intermédiaires commerciaux pour faciliter la mise en relation des agriculteurs périurbains avec les consommateurs de la ville. C'est le cas d'instituts engagés tels l'Instituto Chão et l'Instituto Feira Livre qui proposent à leurs clients des produits bio soumis à une certification alternative OCS. Pour pouvoir les commercialiser, l'Instituto Feira Livre ne dit jamais que la culture est bio mais propose ses produits comme "issus de l'agriculture familiale et sans pesticide " . Les produits qu'on y trouve sont beaucoup moins chers que dans des commerces biologiques plus classiques comme la Feira de Agua Branca.

Pour autant, cette tentative pour accroître la disponibilité de l'offre alimentaire bio et locale ne suffit pas à rééquilibrer les inégalités d'accès pour les populations pauvres. Les consommateurs qui ont recours à ces lieux de vente restent des personnes au capital socio-culturel élevé déjà sensibilisées aux dangers de l'alimentation industrielle. Une logique spatiale de la répartition des instituts Chão et Feira Livre dans la ville contribue à l'expliquer. Le premier se situe à Vila Madalena, un quartier très aisé où les habitants ont des valeurs sociales plutôt libérales, et l'autre est à República, un quartier central en cours de gentrification.

\section{Planifier la ville et l'agriculture face aux conflits fonciers}

17 Il y a 546 exploitations agricoles au sein de la commune de São Paulo, dont 147 dans la zone Nord, 96 dans la zone Est et 307 dans la zone $\operatorname{Sud}^{10}$. Au sein de chaque zone, la situation foncière des producteurs est relativement homogène. Cela s'explique par l'environnement propre à chacune des zones, donnant certaines caractéristiques spécifiques aux exploitations et déterminant leur pérennité.

Dans la zone Nord de São Paulo qui jouxte le Parque Estadual da Cantareira ${ }^{11}$, les exploitations agricoles débordent sur le parc, de la même manière que l'habitat d'autoconstruction. En effet, dans les régions limitrophes situées au Nord et au Sud de la municipalité de São Paulo, les populations les plus défavorisées construisent des 
logements irréguliers et participent à des invasions clandestines qui détruisent des parties de la Mata Atlântica (Santos Ramires et Mello-Théry, 2018). L'habitat d'autoconstruction engendre de multiples problèmes pour la gestion du parc. Peu pérennisé, il cantonne la mise en valeur des espaces dans une logique de court terme. Par manque de structuration, les logements provoquent, entre autres, la stagnation des eaux usées ce qui facilite la transmission de maladies (Pouzenc, 2011). Les agriculteurs présents sur les abords du parc étant des habitants de ces quartiers, ils vivent et produisent dans une grande précarité. Ils possèdent rarement un contrat d'usage et ne sont jamais propriétaires de leur terre, comme D. qui vit et travaille sur une parcelle qu'elle occupe illégalement avec son fils. Alors qu'elle n'a pas d'autre source de revenu, elle est menacée d'expulsion depuis deux ans. Elle n'a pas encore quitté le terrain malgré la demande des autorités.

Dans la zone Est de São Paulo, la situation est d'un autre ordre et l'agriculture se déploie sur des terrains qui ont été dédiés a posteriori à cet usage. Les producteurs, mieux structurés que dans la zone Nord, cultivent des terrains laissés aux agriculteurs dans les années 1980 par un programme de l'État de São Paulo. Les entreprises ont proposé des contrats d'usage aux agriculteurs dans le but de maitriser les invasions illégales de terrains qui ne sont pas constructibles, car déjà occupés par des infrastructures électriques et des canalisations. Par la suite, ce programme n'a connu aucun suivi politique et la plupart des agriculteurs qui ont hérité de leur usage se retrouvent en situation irrégulière car leur contrat n'est plus renouvelé. Ils sont tout juste tolérés par l'entreprise qui ne souhaite pas laisser les espaces vacants, mais sont sans cesse menacés d'expulsion (Giacchè et Porto, 2015).

Illustration 2 - Exploitation agricole dans le district de São Mateus (zone Est) sous les lignes à haute tension d'ElectroPaulo

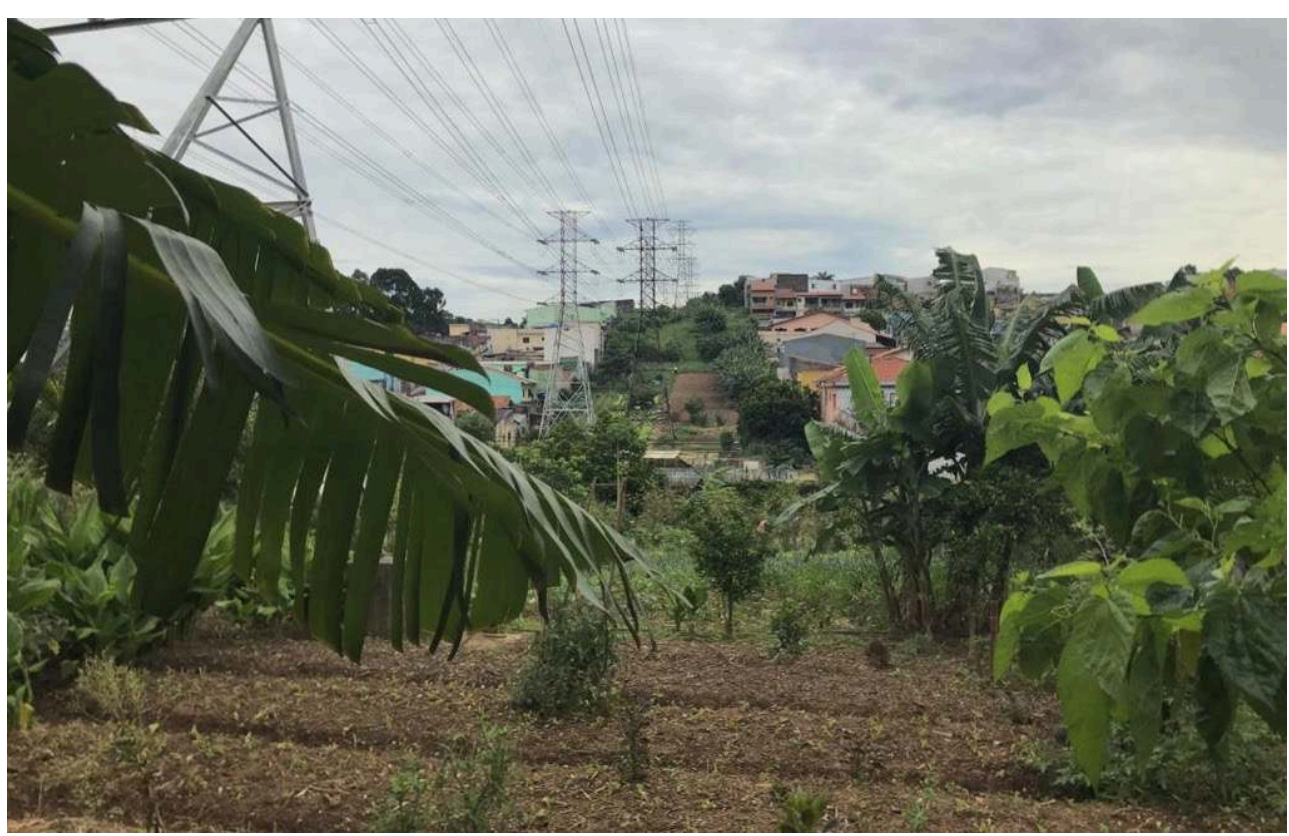

Source : enquêtes de terrain, janvier-mars 2019. Auteur : A. Proust, mars 2019.

Au début des années 2000, la Sous-préfecture de São Mateus ${ }^{12}$ lance un recensement de tous les producteurs qui cultivent dans la zone Est. À la suite de cette identification officielle, les agriculteurs forment de leur plein grès l'Associação dos agricultores da Zona 
Leste (AAZL) qui rassemble actuellement une quarantaine d'agriculteurs. Il s'agit d'une association qui lutte pour le maintien de l'agriculture dans la zone Est et non d'une coopérative de production. La plupart de ses membres cultivent pour leur propre subsistance ou pour distribuer à leurs voisins. Seuls quelques-uns comme Terezinha commercialisent dans le cadre d'une activité économique déclarée.

21 Finalement, la zone Sud apparaît comme la zone la plus rurale et la plus propice à l'activité agricole. Dans cette zone, la plupart des routes ne sont pas asphaltées et les terres sont davantage disponibles pour l'agriculture. On y observe une forme d'agriculture plus pérenne et moins précaire que les deux autres, où les agriculteurs sont davantage propriétaires de leur terrain. La production de la zone Sud est majoritairement conventionnelle - c'est à dire qu'elle utilise des pesticides - et elle est écoulée par des chaînes classiques de distribution agro-alimentaires qui passent par le Ceagesp. Des petits agriculteurs bio de la zone se sont regroupés en 2011 dans la Cooperativa agroecológica dos productores rurais e de água limpa da região Sul ${ }^{13}$ (Cooperapas) dans le but de mettre en commun les moyens de production et de transport des marchandises afin de faciliter la distribution. Il s'agit alors plutôt d'une agriculture familiale biologique calquée sur le modèle de l'agriculture conventionnelle et non d'une activité de subsistance.

Les situations foncières des agriculteurs témoignent des singularités de la zone où se trouve leur exploitation. On remarque que plus la ville et les habitations se rapprochent, plus l'agriculteur est en situation de précarité foncière et économique. Les conditions de vie des squatters de la zone Nord et des producteurs tolérés de la zone Est ne leur permettent pas de gros investissements, ce qui constitue un frein à la recherche de débouchés commerciaux et menace leur environnement proche. À l'autre bout du spectre, les agriculteurs de la zone Sud sont légitimés et mieux organisés ce qui leur laisse la possibilité de s'inscrire dans des circuits de distribution plus amples. Du niveau de reconnaissance de l'agriculteur à l'échelle locale dépend sa capacité à contribuer au système alimentaire et surtout à faire naître une gouvernance alimentaire locale dans les déserts alimentaires de la périphérie. Il s'agit d'une condition nécessaire pour considérer les producteurs périurbains comme les acteurs clés d'une justice alimentaire sur le territoire.

\section{L'agriculture périurbaine comme outil d'autonomisation des acteurs locaux}

\section{La part de l'agriculture périurbaine dans le ravitaillement des quartiers périphériques : modeste mais indispensable}

Il est difficile de quantifier ce que représente l'agriculture périurbaine dans l'approvisionnement global de la métropole, tant les circuits-courts qu'elle engendre sont informels et échappent à ce titre à une évaluation statistique. En revanche, il est possible de montrer le rôle de cette production dans les économies locales et la part qu'elle occupe dans le ravitaillement des quartiers périphériques. La vente en circuits courts peut prendre plusieurs formes, de l'informel local au formel régional. Les plus petits producteurs consomment pour leur propre subsistance et vendent à leurs voisins pour se former une rente supplémentaire. C'est le cas de Sebastiana, qui ne va plus dans les marchés mais commercialise seulement dans sa parcelle depuis que son mari est 
décédé et qu'elle est seule à s'occuper de l'exploitation. L'une de ses clientes déclare acheter tous les légumes et les fruits à l'agricultrice, mais aller chercher le reste viande, féculent - en grande surface. À l'image des autres petits producteurs de la zone Est, Sebastiana assure un approvisionnement au rayonnement limité mais qui se concentre dans des espaces urbains où les produits bio et locaux sont d'une grande rareté. Cela fait des agriculteurs des acteurs clé ouvrant à de nouvelles perspectives de consommation pour des populations habituellement exclues de ces circuits.

Illustration 3a - Récolte pour une cliente de Sebastiana dans le district de São Mateus (zone Est)

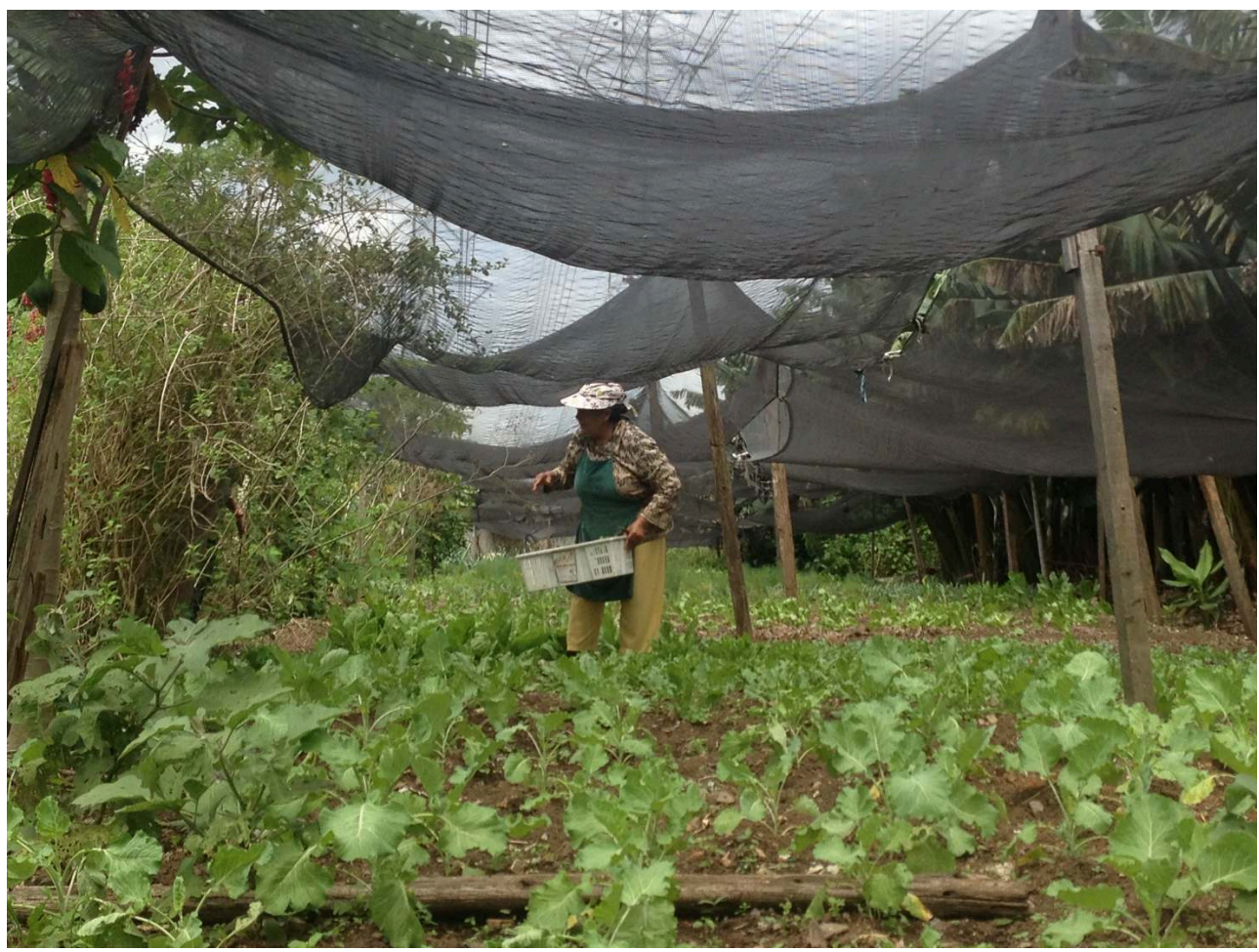

Source : enquêtes de terrain, janvier-mars 2019. Auteur : A. Proust, mars 2019. 
Illustration $3 b$ - Vente directe devant l'exploitation de Sebastiana dans le district de São Mateus (zone Est)

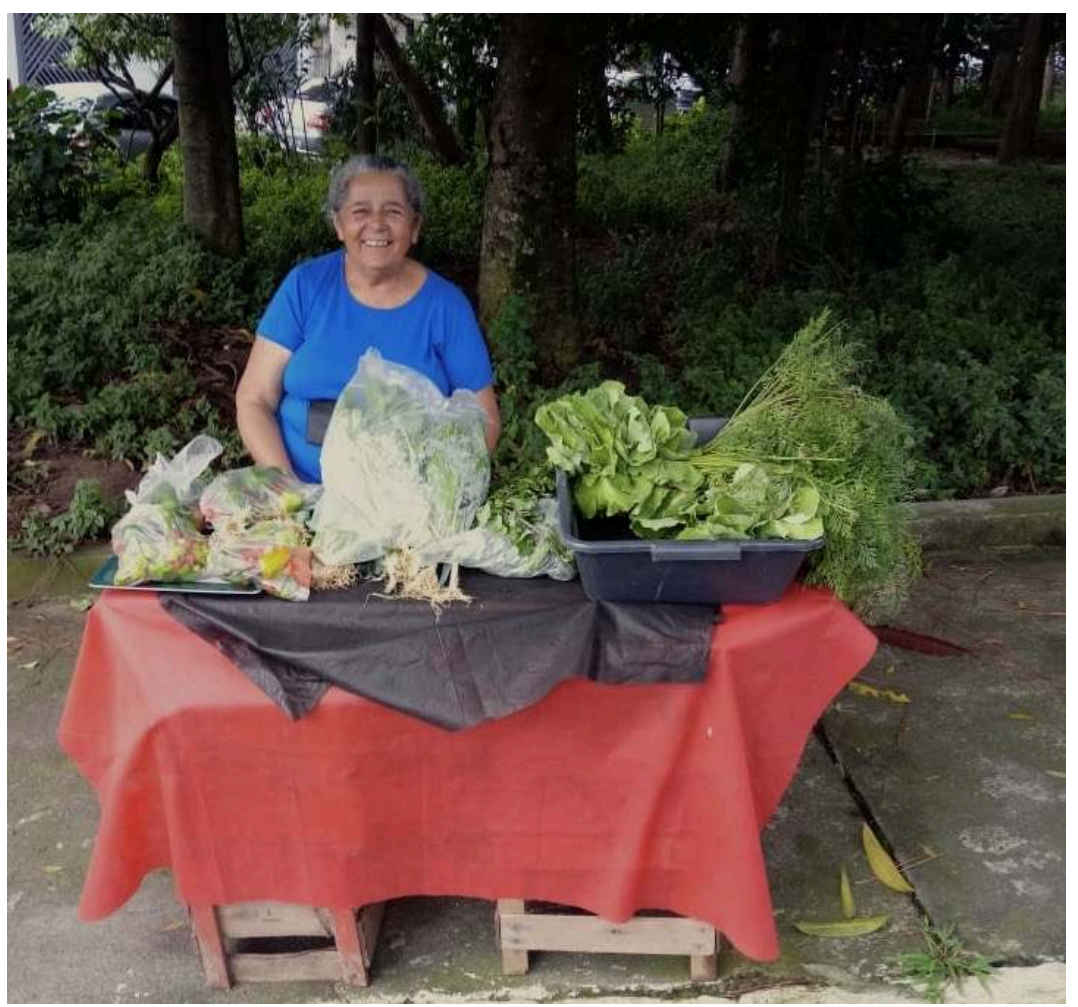

Source : enquêtes de terrain, janvier-mars 2019. Auteur : A. Proust, mars 2019.

Pour autant, bon nombre de personnes qui viennent s'approvisionner chez les agriculteurs ne le font pas parce que cette nourriture est bio et locale, mais parce qu'ils pensent qu'elle sera moins onéreuse qu'une nourriture en grande surface. Les clients négocient régulièrement les prix, ce qui fait partie du jeu pour les producteurs comme Terezinha : «Ici je vends ma salade à 2 reais, alors qu'au marché je la vends à 3. C'est vrai qu'ici on n'a pas à sortir donc c'est forcément moins cher [...]. De toute façon le quartier seul ne me suffit pas. Je dois vendre en dehors ». Terezinha est une des rares agricultrice de la zone Est qui commercialise dans des marchés de plein vent en dehors de son quartier. Elle approvisionne une vingtaine de clients réguliers dans le quartier de São Mateus et vend de façon hebdomadaire à la Feira agroecológica do Tatuapé dans la zone Est. Elle livre aussi ses produits à l'Instituto Feira Livre, ce qui multiplie grandement ses débouchés commerciaux. 
Illustration 4 - Vente de haricots au Marché agroécologique de Tatuapé (zone Est)

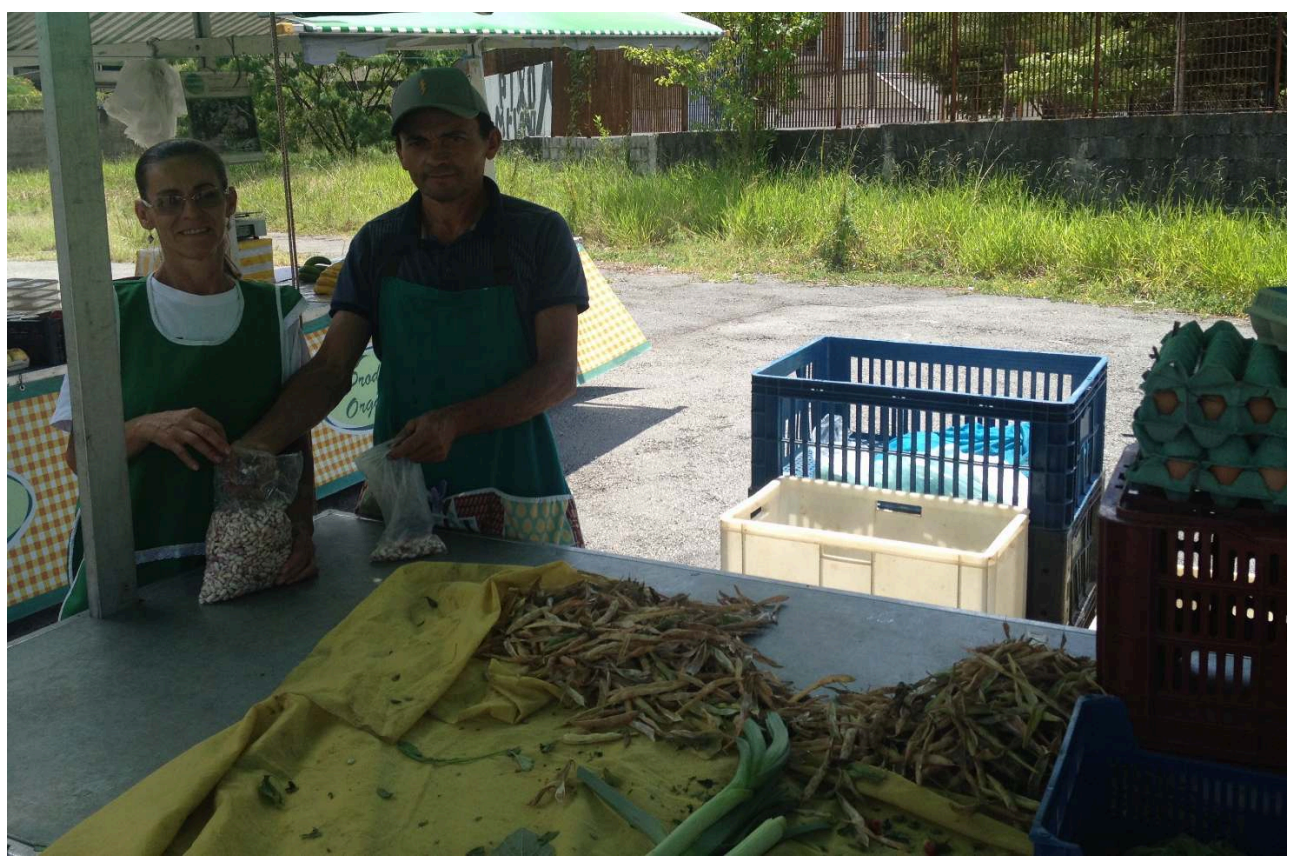

Source : enquêtes de terrain, janvier-mars 2019. Auteur : A. Proust, mars 2019.

Dès lors, les agriculteurs périurbains participent au vivrier marchand qui alimente les espaces les plus défavorisés de la ville, comme la zone Est. Leur production répond aussi de manière qualitative à des enjeux de santé publique, intervenant dans des espaces où les habitants sont incités par la publicité et l'offre commercial à consommer des produits industriels de mauvaise qualité. Il s'agit alors d'un acte politique de contournement des systèmes alimentaires classiques qui implique de nouvelles dynamiques alimentaires urbaines.

\section{Des circuits périphériques de production qui font contrepoids à l'État}

L'agriculture sous-tend toujours une portée symbolique et politique, notamment lorsqu'elle est pratiquée au sein même de la cité dans une perspective de political agriculture (Engelbert, 1953). Ainsi, l'agriculture périurbaine contribue par des actions concrètes aux mécanismes qui font des marges des espaces de lutte politique délaissées des pouvoirs publics. Les populations locales tendent à faire de ces espaces de marge spatio-politique des enclaves d'émancipation. Par leur situation hybride entre ville et campagne, les agriculteurs périurbains sont exclus des logiques agro-alimentaires globales et écartées de certaines politiques publiques qui visent les agriculteurs familiaux ruraux. Ils sont isolés spatialement par une grande proximité à la ville et court-circuités des voies principales de communications routières. Ils sont en décalage avec une vision classique des relations ville-campagne, ce qui conduit à renouveler le regard sur la conceptualisation de la ville et de ses liens avec la campagne (Magrin et Poulot, 2017).

Les producteurs composent avec les moyens qui leur sont donnés et le soutien des habitants de leur propre quartier. Le voisinage s'organise autour d'eux sur la base du volontariat et de la solidarité ce qui donne naissance à des communautés de 
producteurs. Les mutirões ${ }^{14}$ attestent de ces logiques d'entraide, de la même manière que les circuits ultra-courts de distribution au sein des quartiers. À ce titre, l'agriculture périurbaine se distingue comme une pratique d'économie informelle qui offre des perspectives de développement local (Marc, 2011).

Ces mécanismes de construction identitaire sont visibles jusque dans les semences cultivées par les producteurs, qui portent en elles une connotation symbolique et politique. Les denrées produites en périphérie sont issues des régimes anciens de l'alimentation brésilienne, partiellement effacés depuis l'avènement de la cuisine industrielle. C'est le cas des Plantes alimentaires non conventionnelles (PANC), plantes endémiques du Brésil considérées à tort comme des adventices. Celles-ci possèdent de grandes qualités nutritionnelles voire médicinales dans la culture culinaire des régions du Norte est du Nordeste du pays. Actuellement, les populations aisées et cultivées du centre de São Paulo sont en phase de redécouverte de ces plantes qui entrent en connivence avec le recul de la consommation de denrées carnées et les régimes végétariens.

On retrouve aussi beaucoup de tubercules dans les zones périurbaines, principalement le manioc et la patate douce. Ces cultures de plantes et de racines rappellent certains groupes ethniques amérindiens ou australiens qui ont résisté à l'emprise étatique en évitant la monoculture céréalière, facile à contrôler par l'État (Clastres, 1974). Ils lui ont préféré la polyculture du manioc, de la patate douce, de l'igname ou d'autres racines, dont les rendements sont plus difficilement estimables (Scott, 2019). On retrouve cette même logique dans l'agroécologie, une technique agricole qui privilégie la diversité et le mélange des cultivars pour aboutir à un jardin d'apparence anarchique. L'anthropologie politique mêle les exploitations périurbaines à des enclaves de résistance à l'État, à travers un positionnement idéologique en marge des logiques de marché.

\section{Conclusion. Un potentiel en devenir à inclure dans les politiques urbaines}

Une relation plurielle existe entre le politique et l'agriculture à São Paulo. Le manque de politiques publiques, de gestion et de planification précarise les agriculteurs et accentue les pressions foncières en périphérie. En réponse, les logiques de survie des agriculteurs les poussent à adopter des stratégies politiques de contournement des pouvoirs publics et de réadaptation aux systèmes alimentaires classiques. Dans la zone Nord de São Paulo, les producteurs occupent illégalement les versants d'un parc national protégé et leurs exploitations sont extrêmement précaires, tandis que dans la zone Est, la présence des producteurs est tout juste tolérée par les entreprises qui leur prêtent les terrains. À ce titre, les producteurs s'engagent dans l'agriculture en cultivant des terrains qu'ils occupent illégalement. La vente de produits dont les rendements sont difficiles à évaluer (tubercules) et de semences endémiques du Brésil (PANC) relève également d'une émancipation vis-à-vis des systèmes classiques de commercialisation. Ces logiques amenuisent le contrôle exercé par l'État et tendent à créer de la participation politique et de la gouvernance alimentaire à une échelle métropolitaine. 
31 L'échelle métropolitaine semble en mesure de formuler les réponses au défi alimentaire à São Paulo, où l'idéal d'une justice alimentaire guide les actions en faveur de l'agriculture périurbaine. Ces activités agricoles définissent de nouvelles formes d'organisation du système alimentaire métropolitain qui s'appuient sur les acteurs les plus marginalisés: les populations périurbaines et plus particulièrement les producteurs. Celles-ci sont tout autant exclues des systèmes classiques d'approvisionnement que du food movement destiné aux consommateurs possédant un fort capital économique et culturel. Tandis que les agriculteurs périurbains répondent à des enjeux alimentaires majeurs, le rôle joué par les jardiniers urbains est surtout socio-environnemental. Il s'exerce donc une relation ambivalente entre ces deux pratiques. Le jardinage tend à brouiller le rôle de l'agriculture urbaine car il engendre une multiplication de ses usages, mais il peut en même temps lui être favorable en ouvrant la voie à de nouvelles problématiques citoyennes. Ces éléments sont à replacer dans le contexte d'une métropole latino-américaine pluri-millionnaire où l'urbanisation fragmente le tissu paysager et complique toute perspective d'appropriation politique de l'espace. A ce titre, le prisme de l'agriculture urbaine s'inscrit dans un débat sur les modes de gouvernance à São Paulo et mobilise l'alimentation comme l'expression symbolique et identitaire de l'émancipation des populations.

\section{BIBLIOGRAPHIE}

Beischer A., Corbett J., 2016. La justice alimentaire comme réponse à la faim dans les paysages alimentaires canadiens: Comment un projet de glanage communautaire axé sur une praxis de justice alimentaire peut remédier à la dépolitisation de l'insécurité alimentaire. Justice spatiale / spatial justice [En ligne], n 9, p. 1-37. URL: http://www.jssj.org/article/la-justice-alimentairecomme-reponse-a-la-faim-dans-les-paysages-alimentaires-canadiens/

Caldas de Lima E., Jayo M., 2019. Agricultures urbaines à São Paulo: histoire et typologie. Confins Revue franco-brésilienne de géographie / Revista franco-brasilera de geografia [En ligne], nº 39, p. 1-19. URL: http://journals.openedition.org/confins/18683 - DOI: https://journals.openedition.org/ confins $/ 18683$

Chaléard J.-L., 1996. Temps des villes, temps des vivres: l'essor du vivrier marchand en Côte d'Ivoire. Paris, Khartala, 661 p. URL : https://horizon.documentation.ird.fr/exl-doc/pleins_textes/ pleins_textes_7/b_fdi_03_02/010005799.pdf

Clastres P., 1974. La société contre l'État. Recherches d'anthropologie politique. Paris, Collection "Critique », Les éditions de minuit, $186 \mathrm{p}$.

Deverre C., Lamine C., 2010. Les systèmes agroalimentaires alternatifs. Une revue de travaux anglophones en sciences sociales. Economie rurale [En ligne], n 317, p. 56-73. URL: https:// journals.openedition.org/economierurale/2676 
Engelbert E. A., 1968. Agriculture and the Political Process. In Hildreth R.J., Readings in Agricultural Policy. University of Nebraska Press, Lincoln, p. 140-149. URL: https://core.ac.uk/download/pdf/ 7044565.pdf

Giacchè G., Porto L., 2015. Politicas publicas de agricultura urbana e periurbana: uma comparação entre os casos de São e Campinas, Informações econômicas [En ligne], vol. 45, n 6, p. 45-60. URL: http://iea.sp.gov.br/ftpiea/publicacoes/ie/2015/ie-1215.pdf

Hochedez C., Le Gall J., 2016. Justice alimentaire et agriculture. Justice spatiale / spatial justice [En ligne], $n^{\circ}$ 9, p. 1-31. URL: https://www.jssj.org/article/justice-alimentaire-et-agriculture/

Jones N., 2019. Dying to Eat? Black Food Geographies of Slow Violence and Resilience. ACME, An international journal of critical geography [En ligne], vol. 18, n 5, p. 1076-1099. URL: https://acmejournal.org/index.php/acme/article/view/1683/1499

Levasseur P., 2017. Les enjeux socioéconomiques de la transition nutritionnelle au Mexique. Université de Bordeaux - Universidad de Grenada (Espagne), Économies et finances. 303 p. URL: https:// tel.archives-ouvertes.fr/tel-01685611/document

Magrin G., Poulot M., 2017. Conclusion. Les relations villes-campagnes : un prisme d'analyse toujours efficace pour une géographie en renouvellement, in Berger M., Chaléard J.-L., Villes et campagnes en relation. Regards croisés Nords-Suds. Paris, Karthala, p. 277-294.

Marc J-V., 2011. Le jardin créole à Fort-de-France: stratégie de résistance face à la pauvreté ? Vertigo [En ligne], vol. 11, n 1, p.1-16. URL: https://journals.openedition.org/vertigo/10804 - DOI: https://doi.org/10.4000/vertigo.10804

Nagib G., 2016. Agricultura urbana como ativismo na cidade de São Paulo: o caso da Horta das Corujas. São Paulo, Universidade de São Paulo, 434 p. URL: https://www.teses.usp.br/teses/disponiveis/ 8/8136/tde-18082016-124530/publico/2016_GustavoNagib_VOrig.pdf

Paddeu F., 2012. L'agriculture urbaine dans les quartiers défavorisés de la métropole NewYorkaise: la justice alimentaire à l'épreuve de la justice sociale. Vertigo [En ligne], vol. 12, $\mathrm{n}^{\circ} 2$, p. 1-26. URL: https://journals.openedition.org/vertigo/12686

Perrin C., Soulard C.-T., 2014. Vers une gouvernance alimentaire locale reliant ville et agriculture. Le cas de Perpignan. Géocarrefour [En ligne], vol. 89, n 1-2, p. 125-134. URL: https:// journals.openedition.org/geocarrefour/9456

Pouzenc M., 2011. Le défi urbain, in Guibert M., Jean Y., Dynamiques des espaces ruraux dans le monde. Paris, Armand Collin, p. 46-62.

Santos Ramires (dos) J. Z., Mello Théry (de) N. A., 2018. Uso e ocupação do solo em São Paulo, alterações climáticas e os riscos ambientais contemporâneos. Confins [En ligne], n 34, p. 1-23. URL: https://journals.openedition.org/confins/12779 - DOI: https://doi.org/10.4000/confins. 12779

Ramírez M. M., 2015. The Elusive Inclusive: Black Food Geographies and Racialized Food Spaces. Antipode, vol. 47, n 3, p. 748-769. URL: https://onlinelibrary.wiley.com/doi/full/10.1111/anti. 12131

Sbicca J., 2018. Food Justice Now! Deepening the Roots of Social Struggle. Minneapolis, University of Minnesota Press. 288 p.

Scott C. J., 2019. Homo Domesticus. Une histoire profonde des premiers États. Paris, La Découverte, 297 p. 
Tonneau J.-P., Sidersky P., Éloy L., Sabourin E., 2010. Dynamiques et enjeux des agricultures familiales au Brésil. Géoconfluences [En ligne]. URL: http://geoconfluences.ens-lyon.fr/doc/ etpays/Bresil/BresilScient7.htm

Visoni C., Nagib G., 2019. Reappropriating urban space through community gardens in Brazil. Field Actions Science Reports. The journal of field actions [En ligne], Special Issue ${ }^{\circ} 20$, p. 88-91. URL: https://journals.openedition.org/factsreports/5778

Wegner R., Belik W., 2012. Distribuição de hortifruti no Brasil: papel das Centrais de Abastecimento e dos supermercados. Cuadernos de Desarrollo Rural, vol. 9, n 69. p. 194-220. URL: https://www.redalyc.org/pdf/117/11726371005.pdf

\section{NOTES}

1. La Région Métropolitaine de São Paulo est une unité administrative composée de 39 communes dont celle de São Paulo (11,7 millions d'habitants), chacune gouvernée par sa Prefeitura (Mairie). Les échelons de gouvernance de São Paulo sont la Mairie, la Région Métropolitaine et l'État fédéré.

2. Propos recueillis auprès de Hans Christian Temp, responsable financier de l'entreprise Cidade Sem Fome (Ville sans faim) en mars 2019.

3. L'agro-business est un secteur regroupant l'ensemble de la fili ère agro-alimentaire, les entreprises agricoles capitalisées ainsi que les industries de transformation alimentaire. Il représente environ un tiers du Produit Intérieur Brut (PIB) du pays selon le ministère de l'Agriculture au Brésil.

4. URL: https://ligueospontos.prefeitura.sp.gov.br/

5. La Black food geography est un mouvement de géographie radicale qui lie les concepts de la violence émotionnelle à celle de la résilience des populations. Elle étudie comment la violence silencieuse et les traumatiques raciaux sont responsables, bien plus que les choix individuels, de la sur-représentation de maladies nutritionnelles (obésité, diabète) chez les populations racisées. 6. Les deux terrains de recherche (janvier-mars 2019 ; février-mars 2020) nous ont conduit à mener 25 entretiens libres et semi-directifs auprès de différents types d'acteurs : producteurs, jardiniers, personnels associatifs, intermédiaires commerciaux et fonctionnaires de l'État ou de la Mairie - techniciens, agronomes et conseillers en développement rural.

7. Les données des jardins communautaires proviennent du recensement collaboratif de la plateforme Google My Maps, tandis que les exploitations agricoles familiales sont issues du recensement CensoAgro2017 de l'Institut Brésilien de Géographie et de Statistique (IBGE).

8. Carte des foires biologiques au Brésil. URL: https://feirasorganicas.org.br/

9. Propos recueillis auprès d'un employé de l'Instituto Feira Livre le 14 février 2019 à São Paulo.

10. La ville de São Paulo est divisée en 5 grandes zones ou régions administratives qui sont: le centre (Centro), la Zona Oeste (zone Ouest), la Zona Norte (zone Nord), la Zona Sul (zone Sud) et la Zona Leste (zone Est).

11. Il s'agit d'un parc protégé par l'État qui garantit la survie de la Mata Atlântica (Forêt Atlantique). Dans la région Sudeste (Sud-est) du Brésil, ce biome tropical humide est en grande partie dégradé ou menacé de disparition à cause de l'urbanisation de Rio de Janeiro et de São Paulo.

12. Chaque zone de la commune de São Paulo est divisée en différentes Sous-préfectures (Sobprefeitura).

13. Coopérative agroécologique des producteurs ruraux et des eaux propres de la région Sud 14. Un mutirão (mutirões au pl.) est un mot d'origine tupi guarani - langue indigène brésilienne qui signifie «travail en commun». Il s'agit d'une tradition ancienne de solidarité entre les 
membres d'une communauté de voisinage, qui se viennent en aide pour une tâche difficile. Dans ce cas, il s'agit surtout de labourer un champ, en échange de quoi la personne aidée prépare le repas pour tous.

\section{RÉSUMÉS}

Cet article propose une analyse géographique des relations ville campagne à São Paulo, articulée au courant d'études sur la justice alimentaire. Dans un contexte où les politiques publiques sont influencées par les paradigmes de transition, l'enjeu de la relocalisation de l'alimentation semble favorable à une revalorisation de l'agriculture périurbaine. À São Paulo, métropole émergente d'un pays des Suds marqué par une forte polarisation socio-spatiale, les enjeux socioéconomiques, symboliques et politiques d'une reterritorialisation de l'approvisionnement méritent d'être interrogés. Notre hypothèse est que l'agriculture périurbaine, peu soutenue par les politiques publiques, pourrait contribuer à suppléer à un système alimentaire inégalitaire et à créer un «vivrier marchand» qui bénéficierait aux habitants pauvres de la métropole. Ces populations défavorisées vivent dans des marges caractérisées comme des déserts alimentaires et sont les plus touchées par les maladies nutritionnelles (diabète, obésité) causées par la malnutrition.

This article offers a geographical analysis of rural town relations articulate to the studies on food justice in São Paulo. In a context where public policies are influenced by transitional paradigms, the issue of relocating food seems favorable to a revaluation of peri-urban agriculture. In São Paulo, emerging metropolis of a South country marked by strong socio-spatial polarization, the challenges of supply chain's re-territorialisation deserve to be interviewed. They are in the same time socio-economic, health, symbolic and political. Our hypothesis is that peri-urban agriculture, little supported by public policies, nevertheless contributes to supplementing an unequal food system and to creating a "market food crop" which benefits above the poor inhabitants of the metropolis. Those disadvantaged populations live in urban fringes defined as food desert. They are particularly affected by nutritional diseases as diabetes and obesity caused by malnutrition.

\section{INDEX}

Mots-clés : agriculture périurbaine, relation ville campagne, marginalisation socio-spatiale, système métropolitain, gouvernance alimentaire locale, São Paulo

Keywords : peri-urban agriculture, rural town relation, socio-spatial marginalization, metropolitan food system, local food governance, São Paulo

Thèmes : Sur le Champ - Sur le Terrain 
AUTEUR

\section{ANGĖLE PROUST}

Angèle Proust, angele.proust6@gmail.com, est doctorante à l'Université de Paris 1 Panthéon Sorbonne. 